\title{
An Ftp Implementation with Arm Processor and Gprs for Real Time Applications
}

\author{
N. Bala Saidulu ${ }^{1}$, V.Rajasree ${ }^{2}$ \\ ${ }^{1,2}$ (Department of ECE, Ramappa engineering College, Hunter road, Warangal, A.P, India)
}

\begin{abstract}
In this paper, we present the principles of a low-cost Internet-based data processing system. The main heart of the system is an embedded hardware (ARM 9) running with a Linux OS. The embedded device communicates through General Packet Radio Service (GPRS) module, which makes it accessible from anywhere in the world. The GPRS module provides a bidirectional real-time data transfer between the server and client. The proposed system will provide no need for server software and maintenance.

This project can be implemented to any real time application so as to retrieve information from a data source of the server system using GPRS module, and send a file to the remote server through any GPRS enabled mobile device. Based on certain limitations this project may involve in transmitting, receiving a request/file to and fro from server and the mobile device A new approach is introduced to reduce the operational and maintenance costs when dealing with real time or large amount of data. The system is suitable for different embedded applications by attaching several real-time modules through appropriate interfaces.
\end{abstract}

Keywords - ARM, GPRS, GSM, FTP Server ,Data-acquisition, embedded system, interaction, Internet, Linux, real time.

\section{INTRODUCTION}

In the past few years, fixed and mobile networks had a tremendous growth in voice and data processing in good part to the increasing popularity of the Internet. Consequently new data centric applications are emerging and are reaching the general public. At the same time the market is witnessing a remarkable expansion of cellular and mobile technologies leading to demand that data centric applications become available to mobile users. The main objective of this project is to Implement a simple file transfer protocol using GPRS technology and ARM9 processor, while considering various real time issues, such as setting up of the internal clock and adjusting the speeds for sending the request to the server and getting the response at the intended intervals and vice versa, which is enabled for synchronous and asynchronous mode of communication. The General Packet Radio Service (GPRS), a data extension of the mobile telephony standard GSM, is emerging as the first true packet-switched architecture to allow mobile subscribers to benefit from high-speed transmission rates and run many data applications from their mobile terminals. Some applications used remote accessibility are detailed in [1] and [2], which are built to collect and send data through a modem to a server. These systems are well-built to serve the purpose for a specific task, the user cannot interact with the system. Another unidirectional data transfer is presented in [3], which uses the Global System for Mobile Communications (GSM): a popular wireless choice for connectivity between the FTP server unit and clients. A recent work [4] has presented a GPRS solution to the data-acquisition problem for remote areas. An embedded system which is capable of vehicle locating, monitoring, and Tele-metering with GPRS is presented in [5]. A distributed system for long-distance data-collection for the Earth tide gravimeter, information collection about the temperature, humidity, atmospheric pressure, etc., is designed with GPRS using a hard- coded static Internet Protocol (IP) address [6]. These systems use GPRS without concerns about minimizing the cost of data transfer.

\section{CONNECTION METHOD}

When any one needs to access data, must first access the server. To maintain a additional server will also increase the setup costs and maintenance costs of it such as system updates, etc. The closest to this idea is published in [7]. In that system a reliable bidirectional Point-to-Point Protocol (PPP) link used for control and surveillance of real-time applications via a GSM network. In summary there is no effort to minimize the operational costs (which includes the costs to transfer a large amount of data in real-time).

In the proposed system, the GPRS architecture and protocols are compliant with [8]. The generalized structure is shown in the fig.1.The embedded system (FTP server) is configured in such a way that is to be virtually online at all times in a GSM network. Any way the predefined administrative script is executed after booting of the operating system, initiating the GPRS connection software module. A Point-to-point protocol connection is established by a GPRS modem which is capable to operate in the range of operating frequencies at 900/1800/1900 MHz. A PPP daemon (PPPD) is used to control the Point-to-Point Protocol network connections between the client and the embedded system module. The PPPD is 
responsible for setting up the GPRS parameters, such as the connection speed and compression.

\section{CONNECTION ESTABLISHMENT}

To directly access an embedded system, the IP address of the embedded device should be made available to the client side. There are two choices available. A static (hard-coded) IP could be used, or the remote device should initiate a connection by reporting its IP. This choice is quite straightforward and simple. Although the usage cost remains unchanged, it requires a static IP setup by the service provider and involves monthly recurring costs. The static IP is preferred for its simplicity in designing a system; however, its overhead may be impractical. The other choice is to use a dynamic IP assigned through a Dynamic Host Configuration Protocol (DHCP) server of the GSM provider for every connection established. However, this IP needs to be known by any client requesting an access to the embedded server. One solution is to broadcast this IP to a dummy FTP server (where the bulky static information such as image data is also kept). The FTP server is a dummy server and does not require regular software updates or maintenance.

\section{A. Establishing a Direct Communication Link between the Client and the Embedded Device}

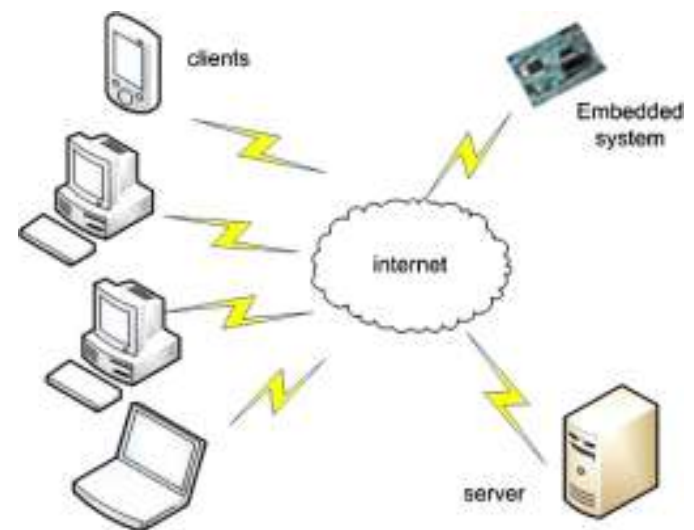

Fig. 1. Generalised diagram for FTP server and clients system

An example embedded system, named temp data log (Fig. 2), can be queried from the FTP server by a simple command. The web browser processes the log index file in the specified folder as default; therefore, a file name is not needed for referencing.

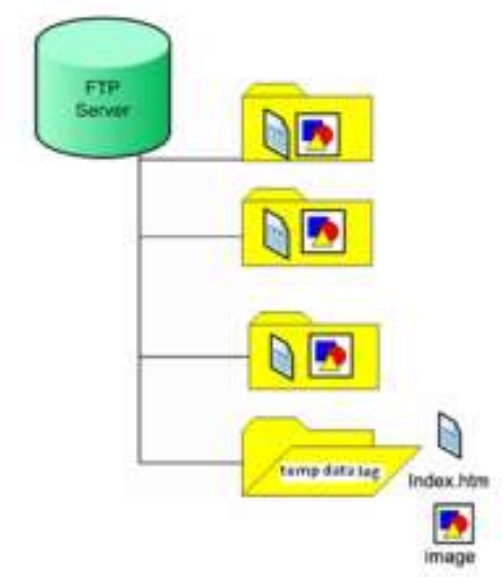

Fig. 2. Folder structure of the FTP server.

B. Data processing in the System

This process is illustrated in Fig. 3. The DHCP approach is more flexible and works better compared with the static approach as a cost-effective solution, despite the necessity for a script running on the embedded server, one-time broadcasting its IP to the FTP server. 


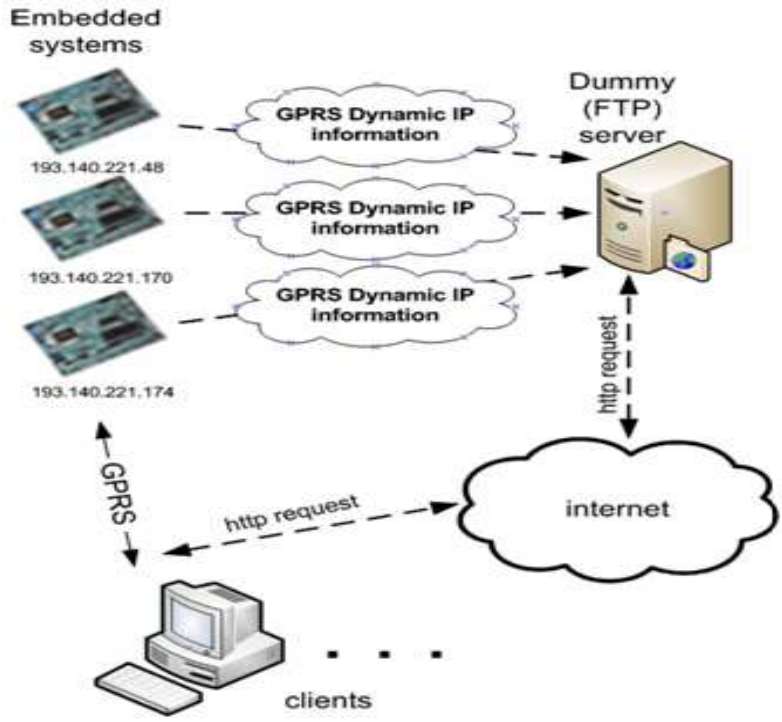

Fig. 3. IP address lookup for the stored IP.

The hypertext file placed on the FTP server by the embedded system and queried by the client is shown in Fig. 4.With this mechanism in place, the embedded system updates it IP information on the FTP server upon every reboot, which causes an IP refresh from the GSM service provider.

Fie Edit View Favorites Tools Help
WELCOME TO THE EMBEDDED SYSTEM WEB ACCESS
Click the hostname below to be directed to its web page
You have requested the IP information for: temp dat log

Fig. 4. Index.htm on the FTP server as viewed by the client

\section{SOFTWARE DESIGN AND HARDWARE DESIGN}

Hardware design

The embedded board used in this work is an ARM9 unit with four serial ports and a parallel port with 64-MB SDRAMonboard removable Flash memory, as shown in Fig. 6. As one of the serial ports is used for coding and debugging purposes. The other serial ports used by the modules are for the following applications

CPU - Samsung S3C2440A 400MHz (Max frequency- 533Mhz )

$\square$ SDRAM

64 MB SDRAM

32-bit Data Bus width

$100 \mathrm{MHz}$ Clock

$\square$ FLASH

128 MB Nand Flash

2 MB Nor Flash, BIOS installed

$\square$ LCD

4 wire resistive Touch Screen Interface

Company : NEC

Panel Type : TFT

Resolution : 320 x 240 RGB

Reflectivity : 7

Illumination : $90 \mathrm{~cd} / \mathrm{m}$

Contrast 10:1 


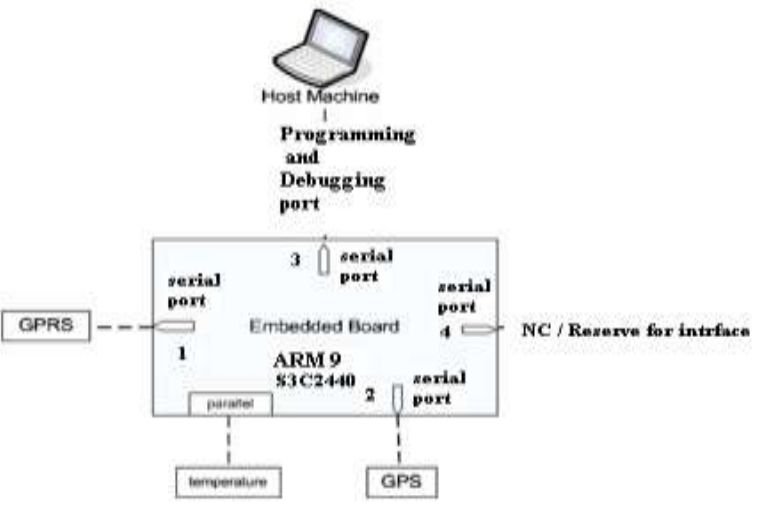

Display Mode : 80:1 Transmissive

Fig. 6. Block diagram of the embedded system with sample devices attached

Applications

1. Vehicle Tracking system using GPS module and GSM modem which is placed in the vehicle ,a periodical communication between the embedded board and the tracking device is done as per the program code, all the data related to location of the vehicle and other information is saved in the FTP server via sms sent by GSM modem in the vehicle for every hour.

2. Room Temperature data logging and saved in the FTP server. For every 15 minutes . if fire accident, the server will send an sms with a message "Fire Accident in the server room" to a pre-defined number , controls the fire extinguisher automatically

3. An IR communication based Burglar alarm which triggers ISR (interrupt services sub-routine program) to execute the alarm and status logged for every 15 minutes. If intrusion occurs send sms "Intrusion" automatically to the pre-defined number and controls the alarm system

4. All the data log files are send to the pre-defined e-mail id for the back up , updates according to the program (for every 6 hours).

All These information can be retrieved by any mobile device by accessing the server for updates at any time, download and upload any amount of data into the server system. Provision for creating a separate folder for individual and access them any time is provided in this project.

Software design

A Linux 2.4 kernel series was selected as required OS for the board

Flow chart

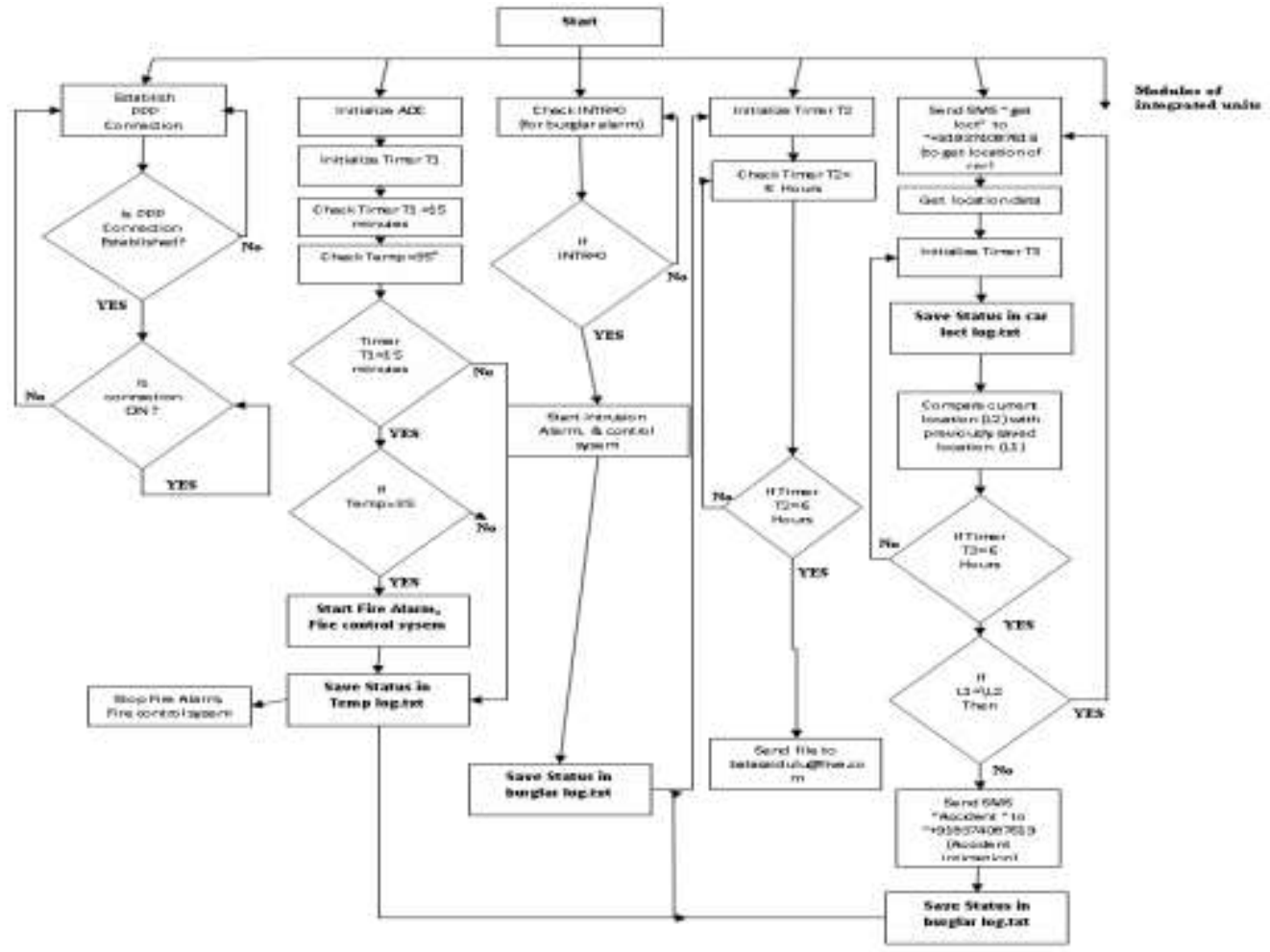




\section{CONCLUSION}

In this paper we present a low- cost FTP server using ARM9 S3C2440 using Linux 2.4 Kernel as operating system, and GPRS module for four different applications. However still more applications can be connected by introducing a multiplexer for any port. Still we need to remember about the power consumption of each device and the embedded system board. Better results can be achieve when it the run in sleep down mode when not in use.

\section{Journal Papers:}

\section{REFERENCES}

[1] W. Kattanek, A. Schreiber, and M. Götze, "A flexible and cost-effective open system platform for smart wireless communication devices," in Proc. ISCE, 2002.

[2] J. E. Marca, C. R. Rindt, and M. G. Mcnally, “The tracer data collection system: Implementation and operational experience," Inst. Transp. Stud- ies, Univ. California, Irvine, CA, Uci-Its-As-Wp-02-2, 2002.

[3] E. Bekiroglu and N. Daldal, "Remote control of an ultrasonic motor by

[4] using a GSM mobile phone," Sens. Actuators A, Phys., vol. 120, no. 2, pp. 536-542, May 17, 2005.

[5] C. Xiaorong, S. Zhan, and G. Zhenhua, "Research on remote data acqui- sition system based on GPRS," in Proc. 8th ICEMI, 2007, pp. 2-20-2-23.

[6] M. A. Al-Taee, O. B. Khader, and N. A. Al-Saber, "Remote monitoring of vehicle diagnostics and location using a smart box with Global Positioning System and General Packet Radio Service,” in Proc. IEEE/ACS AICCSA, May 13-16, 2007, pp. 385388 .

[7] C. Zhang, J. Ge, H. Yu, and X. Zhang, "ET gravimeter data collect- ing system based on GPRS," in Proc. 8th ICEMI, Jul. 18Aug. 16, 2007, pp. 2-86-2-92.

[8] C. E. Lin, C.-C. Li, A.-S. Hou, and C.-C. Wu, "A real-time remote control

[9] architecture using mobile communication," IEEE Trans. Instrum. Meas., vol. 52, no. 4, pp. 997-1003, Aug. 2003.

[10] C. Bettstetter, H.-J. Vögel, and J. Eberspächer, "GSM phase 2+ General Packet Radio Service GPRS: Architecture, protocols, and air interface,"

\section{Books:}

[11] S3C2440A User Manual

[12] Model: DEV 1034.ARM9 Development Board.

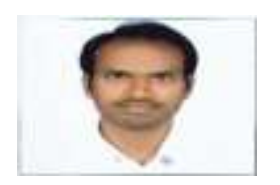

\section{Authors:}

N. Bala Saidulu (M.Tech) Ramappa Engineering College, Hunter Road, Warangal, A.P, India

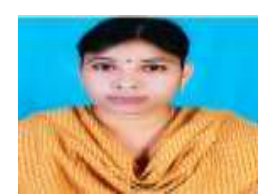

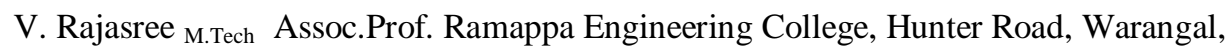
A.P, India 